Journal of Applied AnALysis

Vol. 9, No. 1 (2003), pp. 103-121

\title{
NONEXISTENCE OF GLOBAL SOLUTIONS TO A CLASS OF NONLINEAR DIFFERENTIAL INEQUALITIES AND APPLICATION TO HYPERBOLIC-ELLIPTIC PROBLEMS
}

\author{
N. ALAA and M. GUEDDA \\ Received May 10, 2001 and, in revised form, January 20, 2003
}

\begin{abstract}
We consider the problem$$
u_{t t}+\delta u_{t}+\varepsilon a \Delta u+\varphi\left(\int_{\Omega}|\nabla u|^{2} d x\right) \Delta u \geq f(x, t),
$$

posed in $\Omega \times(0,+\infty)$. Here $\Omega \subset \mathbb{R}^{N}$ is a an open smooth bounded domain and $\varphi$ is like $\varphi(s)=b s^{\gamma}, \gamma>0, a>0$ and $\varepsilon= \pm 1$. We prove, in certain conditions on $f$ and $\varphi$ that there is absence of global solutions. The method of proof relies on a simple analysis of the ordinary inequality of the type

$$
w^{\prime \prime}+\delta w^{\prime} \geq \alpha w+\beta w^{p}
$$
\end{abstract}

It is also shown that a global positive solution, when it exists, must decay at least exponentially.

2000 Mathematics Subject Classification. 35L70, 35J65, 35B40.

Key words and phrases. Nonlinear differential inequalities, hyperbolic and elliptic problems, blow-up, asymptotic behavior of solutions.

ISSN 1425-6908（C) Heldermann Verlag. 


\section{Introduction}

Our initial interest in this paper is the nonexistence of global positive solution to

$$
\left\{\begin{array}{rr}
u_{t t}+\varepsilon a \Delta u+\varphi\left(\int_{\Omega}|\nabla u|^{2} d x\right) \Delta u+\delta u_{t} \geq f(x, t), & \text { on } \Omega \times(0,+\infty), \\
u=0, & \text { in } \partial \Omega \times(0,+\infty),
\end{array}\right.
$$

where $\Omega \subset \mathbb{R}^{N}$ is a bounded domain with smooth boundary $\partial \Omega, a>0$, $\varepsilon= \pm 1, f$ is given function and $\varphi(s)$ is locally Lipschitz function for $s \geq 0$ satisfying

$$
\varphi(s) \geq b s^{\gamma},
$$

where $b$ and $\gamma$ are positive and $\delta$ is a real parameter.

When $\varepsilon=1$ Problem (1.1) is of elliptic type inequality and it is posed in the infinite cylindrical domain $\Omega \times \mathbb{R}_{+}$. If $\varepsilon=-1$ we have, under certain condition, a damped quasilinear hyperbolic inequality.

There is a vast literature for the quasilinear hyperbolic equations with $-\varphi$ instead of $\varphi$. The following model

$$
u_{t t}-\varphi\left(\int_{(0, L)}\left|u_{x}\right|^{2} d x\right) u_{x x}=0
$$

describes, in one dimension, the non-linear vibrations of elastic string. The natural generalization is given by

$$
u_{t t}-\varphi\left(\int_{\Omega}|\nabla u|^{2} d x\right) \Delta u=0
$$

and was studied by Pohozaev [17] in the case where $\varphi$ is a real $C^{1}$ function defined for nonnegative real satisfying $\varphi \geq a_{0}>0$. The author obtained existence and global solutions for analytic initial data. Later Lions [13] formulated the Pohozaev result in abstract context. The work of Ono [16] deals with

$$
u_{t t}+\varphi\left(\left\|A^{1 / 2} u\right\|_{2}\right) A u+\delta u_{t}=|u|^{p} u
$$

where $A=-\Delta, \varphi(s)=a+b s^{\gamma}, a \geq 0, b \geq 0, a+b>0$ and $\gamma>0$. He proved that for $p>2 \gamma$ the local solution is not global if

$$
E(0)<0 \quad\left(\text { resp. } \mathrm{E}(0) \geq 0 \text { and } \int_{\Omega} \mathrm{u}_{0} \mathrm{u}_{1}>0\right),
$$

where $E(0)$ is the initial energy associated to (1.3). 
In [6] Callegari and Manfrin studied the Klein-Gordon equation with the nonlocal nonlinearities

$$
u_{t t}-a\left(\int_{\mathbb{R}^{N}}|\nabla u|^{2} d x\right) \Delta u+\varphi\left(\int_{\mathbb{R}^{N}}|\nabla u|^{2} d x\right) u=0, \quad x \in \mathbb{R}^{N}, t>0,
$$

where $a, \varphi \geq \eta>0$ are $C^{1}(-\theta, \theta)$ functions. The authors proved that if the initial data is sufficiently small, the problem has a unique global regular solution.

We here intend to discuss conditions which assure that the perturbed elliptic-hyperbolic problem (1.1) has no global solution. In particular we obtain a sufficient condition, on the initial data, such that the mean $w(t)=$ $\int_{\Omega} u(x, t) \Phi_{1}(x) d x$ is not global. Here $\Phi_{1}$ is a positive eigenfunction of $-\Delta$ in $H_{0}^{1}(\Omega)$. The key of the proof is to analysis the ordinary differential inequality of the type

$$
w^{\prime \prime}+\delta w^{\prime} \geq \beta w+\alpha w^{p},
$$

where $\delta, \beta \in \mathbb{R}, p>1$ and $\alpha>0$. First we study in Section 2 the global nonexistence of positive solutions to the differential inequalities of the type

$$
w^{\prime \prime}+g\left(w^{\prime}\right) \geq h(w)
$$

As a consequence we prove, in Section 3, the non-global character of solutions to (1.1). In the same manner we investigate the elliptic inequality. We prove that if $u$ is a global nonnegative solution such that $\delta>0$ and $\delta+\lambda_{1} a>1$ then the limit

$$
\lim _{t \rightarrow+\infty} e^{\sqrt{\delta+\lambda_{1} a} t} \int_{\omega} u(x, t) \Phi_{1} d x
$$

is finite. Here $\lambda_{1}$ is the first eigenvalue associated to $\Phi_{1}$. This result is obtained without assuming that $u$ tends to 0 as $t \rightarrow \infty$.

\section{Nonexistence results for differential inequalities}

In this section we collect auxiliary results which will be used later. We investigate here a more general problem of the form

$$
w^{\prime \prime}+g\left(w^{\prime}\right) \geq h(w)
$$

with regard to $w(0)$ and $w^{\prime}(0)$. With the help of methods of ordinary differential inequality, we prove in a certain circumstance that there is absence of global positive solutions to (2.1). The functions $g$ and $h$ are assumed to 
be continuous and satisfy, throughout the paper,

$$
\begin{gathered}
h(r)>0, \quad \text { for all } r>r_{0}, \\
\liminf _{r \rightarrow+\infty} \frac{h(r)}{r^{p}}>0, \quad p>1, \\
g(r) \leq K r, \quad \text { for all } r \in \mathbb{R},
\end{gathered}
$$

where $r_{0}>0, p>1$ and $K$ is a real constant.

In [15], [18] the authors established the nonexistence of global positive solution to a class of differential inequalities of the type $w^{\prime \prime}+A\left|w^{\prime}\right|^{q}+B\left|w^{\prime}\right| \geq$ $C w^{p}, p>q \geq 1$. Here we study a different model. First we have the following

Proposition 2.1. There is no global solution, $w \in C^{2}$, to (2.1) such that

$$
w(0)>r_{0}, \quad w^{\prime}(0) \geq 0 .
$$

Proof. Suppose that a global smooth solution $w$ to (2.1), (2.5) exists. By continuity we have $h(w)>0$ on $\left(0, t_{0}\right), t_{0}$ small. It is easily seen that $w^{\prime}(t)>0$ and then $w(t)>r_{0}$ on $\left(0, t_{0}\right)$. Assume that $w^{\prime}\left(t_{1}\right)=0$ for $t_{1}>0$, then inequality (2.1) cannot be satisfied at $t=t_{1}$. This shows in particular that $w^{\prime}(t)>0$ on $(0,+\infty)$.

Let us prove now that $w(t)$ goes to infinity with $t$. To this end we suppose the contrary; that is $w(t) \rightarrow l>r_{0}$. Hence there exists $\left(t_{n}\right)$ converging to infinity such that $w^{\prime}\left(t_{n}\right)$ tends to 0 . Now multiplying (2.1) by $w$ and integrating the result over $\left(0, t_{n}\right)$ we deduce that

$$
w\left(t_{n}\right) w^{\prime}\left(t_{n}\right)-w(0) w^{\prime}(0)+\frac{K}{2}\left(w^{2}\left(t_{n}\right)-w^{2}(0)\right) \geq \int_{0}^{t_{n}} h(w) w d s,
$$

thanks to (2.4). Therefore $h(w) w \in L^{1}(0,+\infty)$, and then $h(l) l=0$. A contradiction, and then $w(t)$ tends to infinity with $t$. Next the auxiliary function

$$
v:=\frac{1}{2} w^{2}
$$

satisfies the inequality

$$
v^{\prime \prime} \geq w h(w)-K w w^{\prime}+\left(w^{\prime}\right)^{2} .
$$

If $K \leq 0$ we have

$$
v^{\prime \prime} \geq w h(w),
$$

and if $K>0$ we deduce from the Young inequality

$$
v^{\prime \prime} \geq w h(w)-\frac{K^{2}}{4} w^{2} .
$$


Now from (2.3) and the fact that $w$ goes to infinity, we deduce

$$
v^{\prime \prime} \geq \bar{K} w^{p+1}=\bar{K} 2^{(p+1) / 2} v^{(p+1) / 2},
$$

for $t$ large. A simple analysis of this inequality asserts that $v$ is not global since $v$ and $v^{\prime}$ are nonnegative functions and $(p+1) / 2>1$. This contradicts our assumption and finishes the proof.

Remark 2.1. Note that (as inequality (2.1) is autonomous) if there exists $t_{0}>0$ such that $w\left(t_{0}\right)>r_{0}$ and $w^{\prime}\left(t_{0}\right) \geq 0$, the conclusion of the preceding proposition remains true.

Corollary 2.1. Let $p>1, p>q>0, \delta, \beta \in \mathbb{R}$ and $\alpha$ is positive real. There is no global solution $w$ to

$$
w^{\prime \prime}+\delta w^{\prime} \geq \alpha w^{p}-\beta w^{q},
$$

such that

$$
w(0)>\left(\frac{\beta_{+}}{\alpha}\right)^{1 /(p-q)}, \quad w^{\prime}(0) \geq 0,
$$

where $\beta_{+}=\max \{\beta, 0\}$.

Remark 2.2. It is possible to obtain a global solution to (2.6) such that $\beta>0$ and

$$
0<w(0)<\left(\frac{\beta}{\alpha}\right)^{1 /(p-q)}
$$

For example the function

$$
w(t)=\frac{r}{\left(1+e^{s t}\right)^{2 /(p-1)}},
$$

where

$$
r=\left(\frac{\beta}{\alpha}\right)^{1 /(p-q)}, \quad s^{2}=\frac{\alpha(p-1)^{2}}{2(p+1)}\left(\frac{\beta}{\alpha}\right)^{(p-1) /(p-q)}, \quad q=\frac{p+1}{2},
$$

satisfies

$$
w^{\prime \prime}+\frac{2}{p-1} s w^{\prime}=\alpha w^{p}-\beta w^{(p+1) / 2},
$$

on $\mathbb{R}_{+}$. The following result shows that solutions to (2.1) may blow-up at a finite point in the case where $w^{\prime}(0)<0$.

Put

$$
H(r)=\int_{0}^{r} h(s) d s
$$


Proposition 2.2. Assume, in addition, that $r g(r) \geq 0$ for any $r \leq 0$. Then inequality (2.1) has no global solution $w$ such that

$$
\frac{w_{1}^{2}}{2}-H\left(w_{0}\right) \leq-H\left(r_{0}\right)
$$

where

$$
w_{0}=w(0)>r_{0}, \quad w_{1}=w^{\prime}(0)<0 .
$$

Proof. Let $w$ be a global solution satisfying (2.8). Since $w_{1}<0$ the function $w(t)$ is decreasing for small $t$. Assume that there exists $0<t_{0}<\infty$, such that

$$
w\left(t_{0}\right)>r_{0}, \quad w^{\prime}\left(t_{0}\right)=0 .
$$

Using the previous result we deduce that $w$ is not global. Now assume that $w>r_{0}$ on $(0,+\infty)$, and then $w^{\prime}<0$. Integrating $(2.1)$ over $(0,+\infty)$ yields

$$
\int_{0}^{\infty} h(w(s)) d s<+\infty .
$$

Therefore $h(w(\infty))=0$, and then $w(\infty) \leq r_{0}$. Consequently there exists $0<t_{0} \leq+\infty$ such that

$$
w(t)>r_{0}, \quad w^{\prime}(t)<0
$$

on $\left(0, t_{0}\right)$ and

$$
w\left(t_{0}\right)=r_{0}
$$

Next by (2.1) the function

$$
E=\frac{1}{2}\left(w^{\prime}\right)^{2}-H(w)
$$

is strictly decreasing. Hence $E(t)>E\left(t_{0}\right)$, for any $t<t_{0}$. This implies in particular that

$$
\frac{w_{1}^{2}}{2}-H\left(w_{0}\right)>E\left(t_{0}\right)>-H\left(r_{0}\right),
$$

which contradicts (2.8). This finishes the proof.

Corollary 2.2. Let $p>1, p>q>0, \delta \geq 0,1 \geq s>0$ and $\alpha, \beta$ are positive reals. There is no global solution $w$ to

$$
\left\{\begin{array}{l}
w^{\prime \prime}+\delta\left|w^{\prime}\right|^{s-1} w^{\prime} \geq \alpha w^{p}-\beta w^{q}, \\
w(0)=w_{0}, \quad w^{\prime}(0)=w_{1},
\end{array}\right.
$$

such that

$$
\begin{aligned}
& \frac{w_{1}^{2}}{2}-\frac{\alpha}{p+1} w_{0}^{p+1}+\frac{\beta}{q+1} w_{0}^{q+1} \\
& \leq \frac{p-q}{(p+1)(q+1)} \alpha^{-(q+1) /(p-q)} \beta^{(p+1) /(p-q)},
\end{aligned}
$$


where

$$
w_{0}>r_{0}=\left(\frac{\beta}{\alpha}\right)^{1 /(p-q)}, \quad w_{1}<0
$$

We finish this section by simple results concerning the problem

$$
w^{\prime \prime}+\delta w^{\prime}=\alpha e^{\tau t}|w|^{p-1} w+\beta w,
$$

where $\alpha>0, \beta \geq 0$ and $\tau \geq 0$.

When $\delta=1$ a change of variable $y=e^{t}$ transforms this equation into

$$
\frac{d}{d y}\left(y^{2} \frac{d z}{d y}\right)=\alpha y^{\tau}|z|^{p-1} z+\beta z, \quad y \geq 1
$$

which is of the type of the Emden-Fowler equations [4]. First we consider the case $\tau=0$.

Theorem 2.1. Let $\tau=0, p>1, \delta \in \mathbb{R}$ and $\alpha, \beta$ be positive reals. For any $\Gamma \neq 0$ there exists a unique global non trivial solution, $w_{\Gamma}$, to (2.12) such that $w_{\Gamma}(0)=\Gamma$. The function $w_{\Gamma}$ does not change sign and satisfies

$$
\lim _{t \rightarrow+\infty} \frac{w_{\Gamma}^{\prime}(t)}{w_{\Gamma}(t)}=-\frac{\delta+\sqrt{\delta^{2}+4 \beta}}{2} .
$$

Moreover, if in addition $\delta=0$, we have

$$
w_{\Gamma}(t)=\Gamma\left(\frac{2 \sqrt{\beta(p+1)}}{A e^{(p-1) \sqrt{\beta} t}+B}\right)^{2 /(p-1)} e^{\sqrt{\beta} t},
$$

where

$$
\begin{aligned}
& A:=\sqrt{\beta(p+1)+2 \alpha|\Gamma|^{p-1}}+\sqrt{\beta(p+1)} \\
& B:=-\sqrt{\beta(p+1)+2 \alpha|\Gamma|^{p-1}}+\sqrt{\beta(p+1)} .
\end{aligned}
$$

Proof. Since the function $-w$ is also a solution of (2.12) we restrict our considerations to the case $w(0)=\Gamma>0$. Let $\Gamma_{0}$ be any real number and consider

$$
\left\{\begin{array}{l}
w^{\prime \prime}+\delta w^{\prime}=\alpha|w|^{p-1} w+\beta w \\
w(0)=\Gamma, \quad w^{\prime}(0)=\Gamma_{0}
\end{array}\right.
$$

This problem has a unique local solution, $w_{\Gamma, \Gamma_{0}} \in C^{2}\left(\left[0, T_{\Gamma, \Gamma_{0}}\right)\right)$. We shall investigate whether $w_{\Gamma, \Gamma_{0}}$ is global and its properties. It follows from above that if $\Gamma_{0} \geq 0$ then $w_{\Gamma, \Gamma_{0}}$ is not global. Therefore we assume that $\Gamma_{0}<0$. Let us note that if there exists $t_{0} \in\left[0, T_{\Gamma, \Gamma_{0}}\right)$ such that $w_{\Gamma, \Gamma_{0}}\left(t_{0}\right)>0$ and $w_{\Gamma, \Gamma_{0}}^{\prime}\left(t_{0}\right) \geq 0$ then $w_{\Gamma, \Gamma_{0}}$ is not global. Hence if $w_{\Gamma, \Gamma_{0}}$ is global necessarily

$$
w_{\Gamma, \Gamma_{0}}(t)>0, \quad w_{\Gamma, \Gamma_{0}}^{\prime}(t)<0,
$$


for all $t \geq 0$. Using this we can deduce that $w_{\Gamma, \Gamma_{0}}$ and $w_{\Gamma, \Gamma_{0}}^{\prime}$ tend to 0 as $t$ approaches infinity. Next a linearized equation of (2.15), at the origin, is

$$
w^{\prime \prime}+\delta w^{\prime}=\beta w,
$$

and the characteristic equation has the roots $\left(-\delta+\sqrt{\delta^{2}+4 \beta}\right) / 2$ and $-\left(\delta+\sqrt{\delta^{2}+4 \beta}\right) / 2$. From the above consideration we deduce that $(2.15)_{1}$ has exactly one global non trivial solution $w_{\Gamma}$ such that $w_{\Gamma}(0)=\Gamma$. The function $w_{\Gamma}$ is positive and satisfies (2.13). Finally, for $\delta=0$, equation $(2.15)_{1}$ reads

$$
w^{\prime \prime}=\alpha|w|^{p-1} w+\beta w .
$$

Using this and the fact that $w$ and $w^{\prime}$ tend to 0 as $t \rightarrow \infty$, we find

$$
w^{2}=\frac{2 \alpha}{p+1}|w|^{p+1}+\beta w^{2} .
$$

The rest of the proof is done by a simple integration (see [5, p. 360]).

Remark 2.3. For the case where $\beta=0$, we can deduce that if $\delta \geq 0$, any global positive solution satisfies the following estimate

$$
w(t) \leq \frac{\Gamma}{\left(1+\Gamma^{(p-1) / 2} \frac{p-1}{2} \sqrt{\frac{2 \alpha}{p+1} t}\right)^{2 /(p-1)}}=w_{\Gamma}(t),
$$

where $\Gamma=w(0)$. Note that if $\delta=0, w_{\Gamma}$ is the unique global solution to (2.15) such that $w_{\Gamma}(0)=\Gamma$.

Let us now turn to the case $\tau>0$ and $\beta=0$.

Theorem 2.2. Let $\tau, \alpha>0$ and $\delta>-\tau / 2$. Assume that $p>1$, then any global solutions $w$ to (2.12) is monotone and satisfies

$$
\begin{aligned}
0 & <|w| \\
& \leq \frac{\left|w\left(t_{0}\right)\right|}{\left(1+\frac{p-1}{2} \sqrt{\frac{2 \alpha}{p+1}}\left|w\left(t_{0}\right)\right|^{(p-1) / 2}\left(e^{(\tau / 2) t}-e^{(\tau / 2) t_{0}}\right)\right)^{2 /(p-1)}},
\end{aligned}
$$

for all $t \geq t_{0}$, where $t_{0}$ is large.

Proof. It is not difficult to observe that any global non trivial solution does not change sign and it is strictly monotone and bounded. So we may consider only positive global solutions. Let $w$ be a global positive solution. Put $L=\lim _{t \rightarrow \infty} w(t)$. Integrating $(2.12)$ over $(0,+\infty)$ yields $L=0$. Now the function

$$
E(t)=\frac{1}{2} e^{-\tau t} w^{\prime 2}(t)-\frac{\alpha}{p+1} w^{p+1}(t)
$$


satisfies

$$
E^{\prime}(t)=-\left(\frac{\tau}{2}+\delta\right) e^{-\tau t} w^{\prime 2}(t)<0
$$

Next, because $L=0$ and $\liminf w^{\prime}=0$, it follows that $E(t) \geq 0$ and then

$$
-w^{\prime} \geq \sqrt{\frac{2 \alpha}{p+1}} e^{(\tau / 2) t} w^{(p+1) / 2}
$$

Integrating the above inequality we conclude that (2.16) holds. This completes the proof.

For the case $\delta=-\tau / 2$ we have $E=$ const. Hence

Corollary 2.3. Assume that $\delta=-\tau / 2$ and $\beta=0$. Then Problem (2.12) has a unique family of global solutions

$$
w(t)=\frac{w(0)}{\left(1+\frac{p-1}{2} \sqrt{\frac{2 \alpha}{p+1}}|w(0)|^{(p-1) / 2}\left(e^{(\tau / 2) t}-1\right)\right)^{2 /(p-1)}} .
$$

Remark 2.4. Estimate (2.16) remains true if we have greater than in (2.12) instead of equality. Note that $|w| \leq C e^{-[\tau /(p-1)] t}$ for large $t$ and the function

$$
K e^{-[\tau /(p-1)] t},
$$

satisfies (2.12) for any $\delta \in \mathbb{R}$ and any $\beta \geq 0$, where $\tau$ and $K$ are subject to the following conditions

$$
\tau>\frac{p-1}{2}\left(\delta+\sqrt{\delta^{2}+4 \beta}\right)
$$

and

$$
\tau^{2}-(p-1) \delta \tau-(p-1)^{2} \beta=(p-1)^{2} \alpha|K|^{p-1}
$$

Using the function

$$
E_{0}(t)=\frac{1}{2} e^{-\tau t}\left[w^{\prime 2}(t)-\beta w^{2}(t)\right]-\frac{\alpha}{p+1} w^{p+1}(t)
$$

and Proposition 2.2, the preceding theorem is completed by

Proposition 2.3. Let $\tau, \alpha>0, \beta \leq 0$ and $\delta>-\tau / 2$. Assume that $p>1$, then there is no global solution to (2.15) such that

$$
\left(w^{\prime}(0)\right)^{2}<\beta(w(0))^{2}+\frac{2 \alpha}{p+1}|w(0)|^{p+1} .
$$




\section{Nonglobal results for the quasilinear hyperbolic-elliptic inequalities}

The results of Section 2 will allow us to prove the absence of global nonnegative solutions to the following

$$
\left\{\begin{array}{lr}
u_{t t}+\varepsilon a \Delta u+\varphi\left(\int_{\Omega}|\nabla u|^{2} d x\right) \Delta u+\delta u_{t} \geq f(x, t), \\
\text { on } \Omega \times(0, T) \\
u=0, & \text { in } \partial \Omega \times(0, T),
\end{array}\right.
$$

where $\Omega$ is a regular open subset of $\mathbb{R}^{N}, N \geq 1, a>0, \varepsilon= \pm 1$ and $f \in$ $L^{\infty}\left(0, \infty ; L^{2}(\Omega)\right)$. The function $\varphi: \mathbb{R}^{+} \rightarrow \mathbb{R}^{+}$is locally Lipschitz satisfying

$$
\varphi(s) \geq b s^{\gamma}
$$

where $b>0$ and $\gamma>0$.

3.1. The case $\varepsilon=-1$.

Let $\Phi_{1}$ be a positive eigenfunction of

$$
-\Delta \Phi_{1}=\lambda_{1} \Phi_{1}, \quad \Phi_{1_{\mid \partial \Omega}}=0
$$

where $\lambda_{1}$ is the first eigenvalue. We assume that

$$
\left\|\Phi_{1}\right\|_{2}=1 \text {. }
$$

Theorem 3.1. Let $\delta \in \mathbb{R}$ and $\gamma>0$. Assume

$$
\int_{\Omega} f(x, t) \Phi_{1} d x \geq 0
$$

for all $t \geq 0$. Then there exists no global solution to (3.1) such that

$$
\int_{\Omega} u(x, 0) \Phi_{1}(x) d x>\lambda_{1}^{-1 / 2}\left(\frac{a}{b}\right)^{1 / 2 \gamma}, \quad \int_{\Omega} u_{t}(x, 0) \Phi_{1}(x) d x \geq 0 .
$$

Proof. Let $u$ be a global solution. Multiplying equation $(3.1)_{1}$ by $\Phi_{1}$, we get

$$
w^{\prime \prime}+\delta w^{\prime} \geq \lambda_{1} \varphi\left(\int_{\Omega}|\nabla u|^{2} d x\right) w-a \lambda_{1} w
$$

whe re

$$
w(t)=\int_{\Omega} u(x, t) \Phi_{1}(x) d x \quad \text { and } \quad w^{\prime}:=\frac{d w}{d t} .
$$

As $w(0)>0$ one sees $w(t)>0$ on $\left(0, t_{0}\right), t_{0}$ small. On the other hand we have

$$
\varphi\left(\int_{\Omega}|\nabla u|^{2} d x\right) \geq b\left(\int_{\Omega}|\nabla u|^{2} d x\right)^{\gamma}
$$


therefore

$$
\varphi\left(\int_{\Omega}|\nabla u|^{2} d x\right) \geq b \lambda_{1}^{\gamma}\left(\int_{\Omega}|u|^{2} d x\right)^{\gamma} .
$$

Next, using Hölder inequality and (3.3) to deduce that

and then

$$
w^{2}(t) \leq \int_{\Omega} u^{2} d x
$$

$$
\varphi\left(\int_{\Omega}|\nabla u|^{2} d x\right) \geq b \lambda_{1}^{\gamma} w^{2 \gamma}
$$

Substituting this into (3.5) yields

$$
w^{\prime \prime}+\delta w^{\prime} \geq b \lambda_{1}^{\gamma+1} w^{2 \gamma+1}-a \lambda_{1} w,
$$

on $\left[0, t_{0}[\right.$.

Finally, since $\gamma>0, w^{\prime}(0) \geq 0$ and $w(0)$ is larger than $\left(a /\left(b \lambda_{1}^{\gamma}\right)\right)^{1 / 2 \gamma}$, we deduce from Proposition 2.1 that $w$ is not global. A contradiction. This ends the proof.

The following result is an immediate consequence of Corollary 2.2.

Proposition 3.1. Let $\gamma>0$ and $\delta \geq 0$. Let $u$ be a solution to (3.1). Assume

$$
B^{2}+a \lambda_{1} A^{2}-\frac{\lambda_{1}^{\gamma}}{\gamma+1} b A^{2(\gamma+1)} \leq \frac{\gamma}{\gamma+1}\left(\frac{a^{\gamma+1}}{b}\right)^{1 / \gamma}
$$

where

$$
A=\int_{\Omega} u(x, 0) \Phi_{1}(x) d x>\lambda_{1}^{-1 / 2}\left(\frac{a}{b}\right)^{1 / 2 \gamma}
$$

and

Then the function

$$
B=\int_{\Omega} u_{t}(x, 0) \Phi_{1}(x) d x<0 .
$$

is not global.

$$
w(t)=\int_{\Omega} u(x, t) \Phi_{1}(x) d x
$$

Remark 3.1. A similar result can be obtained for the following problems

$$
u_{t t}+\delta u_{t}-a \Delta u-\varphi\left(\int_{\Omega} u^{2} d x\right) u \geq f
$$

and

$$
u_{t t}-a \Delta u+\varphi\left(\int_{\Omega} u^{2} d x\right) \Delta u-\Delta u_{t} \geq f
$$


3.2. The case $\varepsilon=1$.

Here we shall investigate the elliptic inequality

$$
u_{t t}+\delta u_{t}+a \Delta u+\varphi\left(\int_{\Omega}|\nabla u|^{2} d x\right) \Delta u \geq f(x, t)
$$

posed in the half-infinite cylinder

$$
\Sigma:=\Omega \times(0,+\infty)
$$

subject to zero condition on the lateral boundary. Here $\Omega \subset \mathbb{R}^{N}$ is a smooth bounded domain, $a>0$ and $\delta \in \mathbb{R}$. We are concerned with the nonglobal results of solutions to (3.9) and, in the case where the solutions exist, we investigate the asymptotic behaviour as $t$ tends to infinity. A number of papers have appeared in the study of the asymptotic behavior of global solutions. In [20] Véron studied the following

$$
u_{t t}+\Delta u=f(u), \quad \text { in } \Omega \times(0,+\infty),
$$

with Dirichlet condition, where $f$ is a continuous and monotonic function such that $f(0)=0$. The author proved that for any $u_{0} \in L^{p}(\Omega), p \geq 1$, the solution $u$ is bounded, in particular if $p=2, u$ satisfies

$$
\left\|\left.u(t, .)\right|_{L^{\infty}(\Omega)} \leq C t^{-N / 2}\right\| u_{0} \|_{L^{2}(\Omega)}, \quad C=\text { const }>0 .
$$

Later Brada [5] showed that for $F(s) / s^{2} \in L^{2}(-1,1)$, where $F^{\prime}=f$, then

$$
\lim _{t \rightarrow \infty} e^{\sqrt{\lambda_{1}} t} u(t, .)=\alpha \Phi_{1} .
$$

Here we shall give a sufficient condition which asserts the nonexistence of global solutions. As in Section 3.1, $\lambda_{1}$ denotes the first eigenvalue and $\Phi_{1}$ is the positive eigenfunction normalized by $\left\|\Phi_{1}\right\|_{2}=1$. We have the following:

Theorem 3.2. Let $\gamma>0, a>0$ and assume that $\varphi$ and $f$ satisfy (3.2), (3.4). Then Problem (3.9) has no global solution such that

$$
\int_{\Omega} u(x, 0) \Phi_{1}>0
$$

and

$$
\int_{\Omega} u_{t}(x, 0) \Phi_{1} \geq 0
$$

Proof. Let $u$ be a global solution. The function

$$
w(t)=\int_{\Omega} u(x, t) \Phi_{1} d x
$$

is positive and satisfies

$$
w^{\prime \prime}+\delta w^{\prime} \geq \lambda_{1} a w+b \lambda_{1}^{\gamma+1} w^{2 \gamma+1},
$$


in $\left(0, t_{0}\right), t_{0}$ small enough. Arguing as in the proof of Proposition 2.1 one sees that if $w$ exists, it remains positive and satisfies the inequality for all $t>0$. Now as $\gamma>0$ we deduce from Section 2 that $w$ is not global.

The following result will show that a solution may blow-up in the case where

$$
\int_{\Omega} u_{t}(x, 0) \Phi_{1}<0
$$

Theorem 3.3. Let $\gamma>0, a \geq 0$ and $\delta>\max \left(0,1-\lambda_{1} a\right)$. Assume that $\varphi$ and $f$ satisfy (3.2), (3.4). Then there exists no global positive solution to (3.9) such that

$$
\int_{\Omega}\left(\sqrt{\delta+\lambda_{1} a} u(x, 0)+u_{t}(x, 0)\right) \Phi_{1} d x \geq 0 .
$$

Proof. Arguing as in the proof of Theorem 3.2, the mean function satisfies

$$
w^{\prime \prime}+\delta w^{\prime} \geq \lambda_{1} a w+b \lambda_{1}^{\gamma+1}|w|^{p},
$$

where $p:=2 \gamma+1$, subject to the condition

$$
\sqrt{\delta+\lambda_{1} a} w(0)+w^{\prime}(0) \geq 0
$$

The case $w^{\prime}(0) \geq 0$ was treated in the preceding theorem. Suppose now that $w^{\prime}(0)<0$. Put

$$
v(t)=w-c e^{-\sqrt{\delta+\lambda_{1} a} t},
$$

where the constant $c$ satisfies

$$
-\frac{w^{\prime}(0)}{\sqrt{\delta+\lambda_{1} a}}<c<w(0) .
$$

Using inequality (3.12) we infer

$$
v^{\prime \prime}+\delta v^{\prime} \geq \lambda_{1} a v+b \lambda_{1}^{\gamma+1}\left|v+c e^{-\sqrt{\delta+\lambda_{1} a}}\right|^{p} .
$$

As $v(0), v^{\prime}(0)>0$ we deduce from the last inequality that $v$ and $v^{\prime}$ are positive as soon as $v$ exists. Therefore $v$ satisfies

$$
v^{\prime \prime}+\delta v^{\prime} \geq \lambda_{1} a v+b \lambda_{1}^{\gamma+1} v^{p} .
$$

Hence $v$ is not global. This ends the proof.

The following result gives the large time behavior of any possible positive global solution. 
Theorem 3.4. Let $\gamma>0, a \geq 0$ and $\delta>\max \left(0,1-\lambda_{1} a\right)$. Assume that $\varphi$ and $f$ satisfy (3.2), (3.4). Let $u$ be a global positive solution to (3.9), then there exists

such that

$$
0 \leq \alpha_{0} \leq \int_{\Omega} u(x, 0) \Phi_{1} d x
$$

$$
\lim _{t \rightarrow+\infty} e^{\sqrt{\delta+a \lambda_{1}} t} \int_{\Omega} u(x, t) \Phi_{1} d x=\alpha_{0}
$$

Proof. According to the previous result we can see that

$$
\int_{\Omega}\left(\sqrt{\delta+\lambda_{1} a} u(x, t)+u_{t}(x, t)\right) \Phi_{1} d x<0
$$

for all $t \geq 0$. Therefore the function

$$
W(t)=e^{\sqrt{\delta+a \lambda_{1}} t} \int_{\Omega} u(x, t) \Phi_{1} d x
$$

is global, positive and decreasing and then $W$ goes to a finite limit as $t$ tends to infinity.

Corollary 3.1. Assume, in addition, that $a>0, \delta>\max \left(0,1-\lambda_{1} a\right)$ then

$$
\lim _{t \rightarrow \infty} e^{\sqrt{\lambda_{1} a} t} \int_{\Omega} u(x, t) \Phi_{1} d x=0
$$

To complete Theorem 3.3 we have

Theorem 3.5. Let $\gamma>0, a \geq 0$ and $\delta>0$. Assume that $\varphi$ and $f$ satisfy (3.2), (3.4) and

$$
\liminf _{t \rightarrow \infty} \int_{\Omega} f(x, t) \Phi_{1}(x) d x>0 .
$$

Then there is no global positive solution to (3.9).

Proof. Assume that the problem has a global positive solution $u$. Repeated argument of the proof of Theorem 2.1 enables us to see that $w^{\prime}<0, w^{\prime \prime}>0$ and $w$ and $w^{\prime}$ tend to 0 as $t$ approaches infinity. Therefore there exists a sequence $\left(t_{n}\right)_{n}$ converging to infinity such that $w^{\prime \prime}\left(t_{n}\right) \rightarrow 0$ as $n \rightarrow \infty$. Recall that $w$ satisfies

$$
w^{\prime \prime}+\delta w^{\prime} \geq \lambda_{1} a w+b \lambda_{1}^{\gamma+1} w^{2 \gamma+1}+\int_{\Omega} f(x, t) \Phi_{1}(x) d x .
$$

A passage to the limit implies that

$$
0 \geq \lim _{n \rightarrow \infty} \int_{\Omega} f\left(x, t_{n}\right) \Phi_{1}(x) d x
$$

which is absurd. 
The next result is devoted to the general case where $\delta \in \mathbb{R}$.

Theorem 3.6. Let $\gamma>0, a \geq 0$ and $\delta \in \mathbb{R}$. Assume that $\varphi$ and $f$ satisfy (3.2), (3.4). Then there exists no global solution to (3.9) such that

$$
\int_{\Omega}\left(\delta+\sqrt{\delta^{2}+4 \lambda_{1} a} u(x, 0)+2 u_{t}(x, 0)\right) \Phi_{1} d x>0 .
$$

Proof. Let $\mathrm{u}$ be a solution to (3.9). Arguing as in the proof of Theorem 3.1 , the function $w(t)=\int_{\Omega} u(x, t) \Phi_{1}(x) d x$ satisfies

$$
w^{\prime \prime}+\delta w^{\prime} \geq \lambda_{1} a w+b \lambda_{1}^{1+\gamma}|w|^{p} .
$$

Let $k=\left(-\delta+\sqrt{\delta^{2}+4 \lambda_{1} a}\right) / 2$ and $\eta=\sqrt{\delta^{2}+4 \lambda_{1} a}$, while using the change of variable $w(t)=\exp (k t) v(t)$ one obtain the following

$$
v^{\prime \prime}+\eta v^{\prime} \geq b \lambda_{1}^{1+\gamma} \exp ((p-1) k t)|v|^{p} .
$$

Now we consider $\theta \in C_{0}^{\infty}\left(\left[0,+\infty[), \theta \geq 0\right.\right.$ such that $\theta(0)=1, \theta^{\prime}(0)=0$ and

$$
\int_{0}^{\infty}\left|\theta^{\prime \prime}\right|^{q} \theta^{1-q} d t+\int_{0}^{\infty}\left|\theta^{\prime}\right|^{q} \theta^{1-q} d t<\infty
$$

where $q=p /(p-1)$. If one multiplies (3.14) by $\theta$ and integrates by parts, one gets

$$
\begin{aligned}
& \eta v(0)+v^{\prime}(0)+b \lambda_{1}^{1+\gamma} \int_{0}^{+\infty} \exp ((p-1) k t)|v|^{p} \theta(t) d t \\
& \leq M \int_{0}^{\infty}|v|\left(\left|\theta^{\prime \prime}\right|+\left|\theta^{\prime}\right|\right) d t,
\end{aligned}
$$

where $M=\max \{1, \eta\}$. Making use of the Young inequality, one finds, for $\varepsilon>0$,

$$
\begin{aligned}
\int_{0}^{\infty}|v|\left(\left|\theta^{\prime \prime}\right|+\left|\theta^{\prime}\right|\right) d t \leq & \varepsilon \int_{0}^{\infty} \exp ((p-1) k t)|v|^{p} \theta d t \\
& +C_{\varepsilon} \int_{0}^{\infty} \exp (-k t)\left(\left|\theta^{\prime}\right|^{q}+\left|\theta^{\prime \prime}\right|^{q}\right) \theta^{1-q} d t .
\end{aligned}
$$

Next we take $\varepsilon=b \lambda_{1}^{1+\gamma} / M$, to deduce, for some positive constant $C$

$$
\eta v(0)+v^{\prime}(0) \leq C \int_{0}^{+\infty} \exp (-k t)\left(\left|\theta^{\prime}\right|^{q}+\left|\theta^{\prime \prime}\right|^{q}\right) \theta^{1-q}(t) d t,
$$


thanks to (3.16). Now for $T>0$ fixed, choosing $\theta(t)=\theta_{0}^{r}(t / T)$ where $\theta_{0} \in C_{0}^{\infty}\left(\left[0,+\infty[), 0 \leq \theta_{0} \leq 1, \theta_{0}(t)=1\right.\right.$ for $0 \leq t \leq 1 / 2$ and $\theta_{0}(t)=0$ for $t \geq 1$, and where $r \geq 2 q$ such that condition (3.15) holds. Using estimate (3.17), we easily obtain

$$
\begin{aligned}
\eta v(0)+v^{\prime}(0) \leq & C T^{1-2 q} \exp \left(-\frac{k T}{2}\right) \int_{1 / 2}^{1}\left|\theta^{\prime \prime}\right|^{q} \theta^{1-q} d t \\
& +C T^{1-q} \exp \left(-\frac{k T}{2}\right) \int_{1 / 2}^{1}\left|\theta^{\prime}\right|^{q} \theta^{1-q} d t
\end{aligned}
$$

and then

$$
\eta v(0)+v^{\prime}(0) \leq \bar{C}\left\{T^{1-2 q} \exp \left(-\frac{k T}{2}\right)+T^{1-q} \exp \left(-\frac{k T}{2}\right)\right\}, \quad \bar{C}>0 .
$$

We may let $T \rightarrow \infty$ in the above inequality to deduce that

$$
\eta v(0)+v^{\prime}(0) \leq 0
$$

which contradicts hypothesis (3.13). This ends the proof.

Remark 3.2. The results of Section 2 can also be used to obtain a family of global and nonglobal "explicit" solutions to

$$
u_{t t}+\delta u_{t}+\left(\int_{\Omega}|\nabla u|^{2} d x\right)^{\gamma} \Delta u-\beta u \geq \lambda|u|^{p-2} u, \quad \text { in } \Sigma,
$$

with Dirichlet boundary condition, where $\lambda, \beta>0$ and $p=2(\gamma+1)>1$. Here we look for solutions having the form

$$
u(x, t)=w(t) v(x) .
$$

In fact if we substitute (3.19) into (3.18), we obtain the following inequality

$$
v\left(w^{\prime \prime}+\delta w^{\prime}-\beta w\right) \geq|w|^{2 \gamma} w\left[-\left(\int_{\Omega}|\nabla v|^{2} d x\right)^{\gamma} \Delta v+\lambda|v|^{2 \gamma} v\right] .
$$

Therefore it is sufficient to demand to the required functions $w$ and $v$ to satisfy

$$
w^{\prime \prime}+\delta w^{\prime} \geq \kappa w^{2 \gamma+1}+\beta w,
$$

and

$$
-\left(\int_{\Omega}|\nabla v|^{2} d x\right)^{\gamma} \Delta v+\lambda|v|^{2 \gamma} v=\kappa v, v \geq 0
$$

where $\kappa>0$.

We have 
Theorem 3.7. Let $\lambda, \kappa>0$ and $\gamma>0$ such that $(N-2) \gamma<2$. Let $v_{0} \in H_{0}^{1}(\Omega)$ be a non trivial solution to (3.21). Then for any $a>0, b<0$ satisfying

$$
b^{2}+\delta a b-\beta a^{2} \geq \kappa a^{2(\gamma+1)},
$$

the function

$$
U(x, t)=a e^{(b / a) t} v_{0}(x),
$$

is a non trivial solution to (3.18).

Proof. First we show that (3.21) has a non trivial solution. To this direction we consider the problem

$$
\inf \left\{\left(\int_{\Omega}|\nabla v|^{2}\right)^{\gamma+1} d x+\lambda \int_{\Omega}|v|^{2(\gamma+1)} d x ; v \in H_{0}^{1}(\Omega), \int_{\Omega}|v|^{2}=1\right\} .
$$

As $(N-2)(2 \gamma+1)<N+2$, there exists $v_{0} \in H_{0}^{1}(\Omega)$ which realizes the infimum; it is nonnegative and satisfies the Euler-Lagrange equation (3.21). Next we look for solution to (3.20) in the form $w(t)=a e^{(b / a) t}, a>0, b<0$. Insertion into (3.20) yields

$$
\left(\frac{b^{2}}{a}+\delta b-\beta a\right) e^{(b / a) t} \geq \kappa a^{2 \gamma+1} e^{(2 \gamma+1)(b / a) t} .
$$

This leads to the sufficient condition (3.22).

Remark 3.3. If we have equality in (3.18) instead of inequality, it is clear that $w$ would satisfy

$$
w^{\prime \prime}+\delta w^{\prime}=\kappa w^{2 \gamma+1}+\beta w .
$$

Then, for $\delta=0$, the function

$$
U(x, t)= \begin{cases}\frac{\Gamma v_{0}(x)}{\left(1+\gamma \Gamma \gamma \sqrt{\frac{\kappa}{\gamma}} t\right)^{1 / \gamma}}, & \text { if } \beta=0, \\ \Gamma\left(\frac{2 \sqrt{\beta(\gamma+1)}}{A e^{2 \gamma \sqrt{\beta} t}+B}\right)^{1 / \gamma} e^{\sqrt{\beta} t} v_{0}(x), & \text { for } \beta>0,\end{cases}
$$

where

$$
\begin{aligned}
& A:=\sqrt{\beta(\gamma+1)+\alpha|\Gamma|^{2 \gamma}}+\sqrt{\beta(\gamma+1)} \\
& B:=-\sqrt{\beta(\gamma+1)+\alpha|\Gamma|^{2 \gamma}}+\sqrt{\beta(\gamma+1)},
\end{aligned}
$$

satisfies (3.18). 
Acknowledgments. This work is partially supported by French-Morocco scientific cooperation project "Action-Intégrée" No 182/MA/99. The second author is grateful for a partial support from the Department of Mathematics of Faculty of Sciences and Technique, FST Marrakech Maroc, during his visit there.

\section{References}

[1] Ablowitz, M. J., Segur, H., Exact linearization of a Painlevé transcendent, Phys. Rev. Lett. 38 (1977), 1103-1106.

[2] D'Ancona, P., Spagnolo, S., A class of nonlinear hyperbolic problems with global solutions, Arch. Rational Mech. Anal. 124 (1993), 219-281.

[3] Ball, J. M., Remarks on blow-up and nonexistence theorems for non linear evolution equations, Quart. J. Math. Oxford Ser. (2) 28 (1977), 473-486.

[4] Bellman, R., Stability Theory of Differential Equations, McGraw-Hill, New York, 1953.

[5] Brada, A., Comportement asymptotique de solutions d'équations elliptiques semilinéares dans un cylindre, Asymptotic Anal. 10 (1995), 335-366.

[6] Callegari, E., Manfrin, R., Global small solutions to Klein Gordon type equations with non-local non-linearities, Nonlinear Anal. 38 (1999), 505-526.

[7] Eloulaimi, R., Guedda, M., Nonexistence of global solutions of nonlinear wave equations, Portugal. Math. 58(4) (2001), 449-460.

[8] Gmira, A., Guedda, M., Classification of solutions to a class of nonlinear differential equations, Int. J. Differ. Equ. Appl. 1(2) (2000), 223-238.

[9] Greenberg, J. M., Hu, S. C., The initial value problem for stretched string, Quart. Appl. Math. 38 (1980), 280-311.

[10] Guedda, M., Kirane, M., Nonexistence of global solutions to nonlinear hyperbolic inequalities with a singularity, Preprint LAMFA (2000), Université de Picardie Jules Verne, France.

[11] Hastings, S. P., McLeod, J. B., A boundary value problem associated with the second Painlevé transcendent and the Korteweg-de Vries equation, Arch. Rational Mech. Anal. 73(1) (1980), 31-51.

[12] Guedda, M., Labani, H., Nonexistence of global solutions to a class of nonlinear wave equations with dynamic boundary conditions, Bull. Belg. Math. Soc. Simon Stevin 9(1) (2002), 39-46.

[13] Lions, J. L., On some questions in boundary value problems of mathematical physics. Contemporary developments in continuum mechanics and partial differential equations, Proc. Internat. Sympos., Inst. Mat., Univ. Fed. Rio de Janeiro, Rio de Janeiro, 1977, pp. 284-346, North-Holland Math. Stud. 30, North-Holland, Amsterdam-New York, 1978.

[14] Miura, R. M., The Korteweg - de Vries equation; a survey of results, SIAM Rev. 18 (1976), 412-459.

[15] Oleinik, O., Some Asymptotic Problems in the Theory of Partial Differential Equations, Lincei Lectures, Cambridge University Press, Cambridge, 1996.

[16] Ono, K., Global existence, decay, and blowup of solutions for some mildly degenerate nonlinear Kirchhoff strings, J. Differential Equations, 137(2) (1997), 273-301.

[17] Pohozaev, S. I., On a class of quasilinear hyperbolic equations, Mat. Sb. (N. S.) 95 (1975), 152-166.

[18] Souplet, Ph., Nonexistence of global solutions to some differential inequalities of the second order and applications, Portugal. Math. 52(3) (1995), 289-299. 
[19] Vasconcellos, C. F., Teixeira, L. M., Strong solution and exponential decay for a nonlinear hyperbolic equation, Appl. Anal. 51(1-4) (1993), 155-173.

[20] Véron, L., Coercivité et propriété régularisante de semi-groupes non linéaires dans les espaces de Banach, Publ. Math. Fac. Sci. Besançon 3 (1976-1977).

N. AlaA

Faculté des Sciences

ET Techniques

Université CADI AYyAD

BP 618 MARRAKECH

MAROC
M. GuedDA

LAMFA, CNRS UMR 6140

Université de Picardie Jules Verne

FACUlté de Mathématiques

ET D'INFORMATIQUE

33, Rue Saint-Leu 80039 Amiens

FRANCE 